\title{
Size does not always matter: Experimental evidence to inform the open-ocean microbial gardening hypothesis
}

\author{
emma cavan ${ }^{1}$, So Kawaguchi², and Philip Boyd ${ }^{3}$ \\ ${ }^{1}$ Imperial College London Department of Life Sciences \\ ${ }^{2}$ Australian Antarctic Division \\ ${ }^{3}$ University of Tasmania College of Sciences and Engineering
}

June 16, 2020

\begin{abstract}
1. Detritivores need to up-cycle their food to increase its nutritional value. One method is to fragment detritus promoting the colonisation of nutrient-rich microbes, which consumers then ingest. This is known as microbial gardening. Observations and numerical models of the detritus-dominated ocean mesopelagic zone have suggested microbial gardening by zooplankton is fundamental process in the ocean organic carbon cycle, as it leads to increased respiration of carbon-rich detritus. However, no experimental evidence exists to prove microbial respiration is higher on smaller, fragmented detrital particles. 2. Using aquariareared Antarctic krill faecal pellets we showed fragmentation increased microbial particulate organic carbon (POC) turnover by $70 \%$, but only on brown faecal pellets of low nutritional value. Microbial POC turnover on un-and fragmented green faecal pellets of higher nutritional value was equal. Thus we find particle size alone is not enough to determine microbial activity, and the nutritional value and age of the particle are important. 3. We estimate mesopelagic zooplankton can potentially increase the proportion of essential nutrients (e.g. unsaturated fatty acids) in their food by at least $11 \%$. In addition we propose 'communal gardening' may occur whereby other mesopelagic organisms consume the particle and microbes gardened by a neighbouring detritivore. 4. Increases in microbial turnover of detrital POC reduces the sink of organic carbon in the ocean. Thus microbial gardening should be represented in models forecasting the future carbon cycle. Model parameterisations will require further understanding of the energetic gains to zooplankton communities, how microbial gardening influences other sinking particles such as detrital aggregates, and the relative importance of biological (i.e. particle lability, size and age) vs. physical (i.e. temperature and oxygen) constraints on gardening.
\end{abstract}

\section{Hosted file}

Main_article.pdf available at https://authorea.com/users/333862/articles/459938-size-doesnot-always-matter-experimental-evidence-to-inform-the-open-ocean-microbial-gardeninghypothesis 